\title{
Relativistic and Radiative Energy Shifts for Rydberg States
}

\author{
Ulrich D. Jentschura, ${ }^{1,2}$ Eric-Olivier Le Bigot,${ }^{3}$ Jörg Evers, ${ }^{2}$ Peter J. Mohr, ${ }^{4}$ and Christoph H. Keitel ${ }^{1,2}$ \\ ${ }^{1}$ Max-Planck-Institut für Kernphysik, Saupfercheckweg 1, 69117 Heidelberg, Germany \\ ${ }^{2}$ Theoretische Quantendynamik, Physikalisches Institut, Universität Freiburg, 79104 Freiburg im Breisgau, Germany \\ ${ }^{3}$ Laboratoire Kastler Brossel, École Normale Supérieure et Université Pierre et Marie Curie, Case 74, 75005 Paris, France \\ ${ }^{4}$ National Institute of Standards and Technology, Mail Stop 8401, Gaithersburg, MD 20899-8401, USA
}

\begin{abstract}
We investigate relativistic and quantum electrodynamic effects for highly-excited bound states in hydrogenlike systems (Rydberg states). In particular, hydrogenic one-loop Bethe logarithms are calculated for all circular states $(l=n-1)$ in the range $20 \leq n \leq 60$ and successfully compared to an existing asymptotic expansion for large principal quantum number $n$. We provide accurate expansions of the Bethe logarithm for large values of $n$, for $S, P$ and circular Rydberg states. These three expansions are expected to give any Bethe logarithms for principal quantum number $n>20$ to an accuracy of five to seven decimal digits, within the specified manifolds of atomic states. Within the numerical accuracy, the results constitute unified, general formulas for quantum electrodynamic corrections whose validity is not restricted to a single atomic state. The results are relevant for accurate predictions of radiative shifts of Rydberg states and for the description of the recently investigated laser-dressed Lamb shift, which is observable in a strong coherent-wave light field.
\end{abstract}

PACS numbers: 12.20.Ds, 31.30.Jv, 31.15.-p, 11.10.Jj

\section{INTRODUCTION}

Circular Rydberg states $(n-1=l=|m|)$ have attracted attention in the past two decades, in part because the transitions among these states are resonant with typical modes of microwave cavities. A convenient mechanism for the preparation of these states has been described in 1, 2, 3]. When coupled to the modes of a microwave cavity, the metastable Rydberg atoms undergo Rabi oscillations, leading to a direct verification of the concept of field quantization [4]. The coupling of the resonant cavity to the Rydberg atom can be used for the generation of entangled Einstein-Podolsky-Rosen pairs of atoms [ $[$ ]. A further field of interest has been the theoretical simulation of atomic non-dispersive wave packets, which can be realized with the "assistance" of a background laser field with a specific "Kepler" frequency (see, e.g., [6, 7, [8]).

This partial list of applications is supplemented here by an investigation of relativistic and radiative effects in highlyexcited hydrogenic states, without any consideration of the additional interaction with a cavity. The electron density of circular Rydberg states $(n-1=l=|m|)$ resembles Kepler circular orbits (see Fig. 11). However, the characteristic shape of wave function is restricted to the maximum- $|m|$ subcomponent (see also Fig. 1). For states with nonmaximal $|m|<l$, the shape of the electron density is manifestly different. For all circular Rydberg states, the wave function is spread out considerably in comparison to lower states, on a length scale of $\langle r\rangle \sim$ const. $\times n^{2} a_{\text {Bohr }}$ (see Ref. [9]), where $r$ is the radial coordinate, $a_{\mathrm{Bohr}}$ is the Bohr radius and $n$ is the (high) principal quantum number. Relativistic and radiative effects in Rydberg atoms allow for a simultaneous perspective on four different physical regimes: relativistic and QED radiative effects in the transition in the borderline region between classical and quantum physics.

In addition to the aforementioned reasons for studying Rydberg atoms, the current paper is also inspired by a recently completed analysis of radiative corrections for laser-dressed states [10, 11, 12]. In [10, 12], an experimental setup based on the hydrogen $1 S-2 P_{j}(j=1 / 2,3 / 2)$ transition is discussed in detail. A coherent-wave light source with the appropriate frequency has recently become available [13]. Because the $1 S$ ground state is fully stable against radiative (spontaneous) decay, the incoherent atomic fluorescence (in the case of the $1 S-2 P_{j}$ transition) is described to excellent accuracy by the Mollow spectrum [14]. A further promising setup for studying the laser-dressed Lamb shift would be provided by a transition from the metastable $2 S$ state to a high- $n$ (Rydberg) $P$ state. Rydberg states have a small radiative decay width, which would facilitate the observation of the predicted dynamic corrections to the Lamb shift [10, 15]. Furthermore, the frequency required for the excitation process $2 S \Leftrightarrow n P_{j}($ high $n$ ) is smaller and therefore a priori less problematic to realize experimentally than the $1 S-2 P_{j}$ transition. In the formalism of [10, 11, 12], the self-energy correction analyzed here contributes to the Lamb shift of "bare" atomic levels; this contribution is an essential part of the laser-dressed Lamb shift, which may be observed in incoherent light scattering by hydrogenlike atoms.

Another motivation for the current work is to find actual values of the Bethe logarithms for highly-excited states, since the Bethe logarithm gives a significant contribution to radiative level shifts. This is true of hydrogenlike, as well as heliumlike [16, 17, 18] and lithiumlike 19] systems. One of the most extensive systematic studies [20] of hydrogenic (one-loop) Bethe logarithms currently available in the literature extends only up to $n=20$, and only approximate values are available for higher excited circular Rydberg states [23]. Furthermore, it is of interest to study 


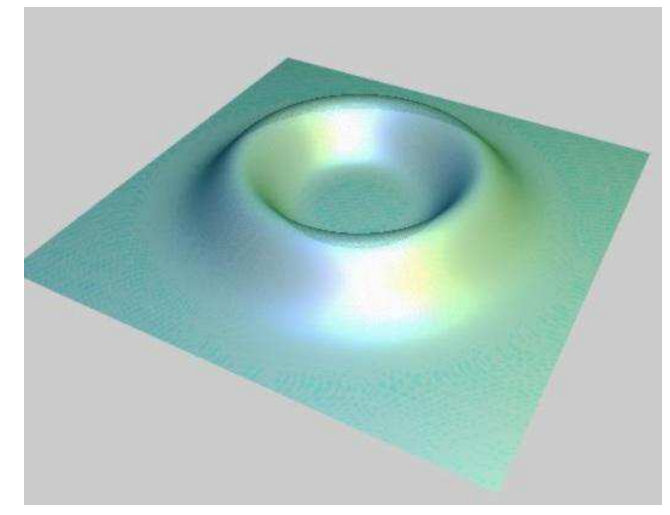

(a)

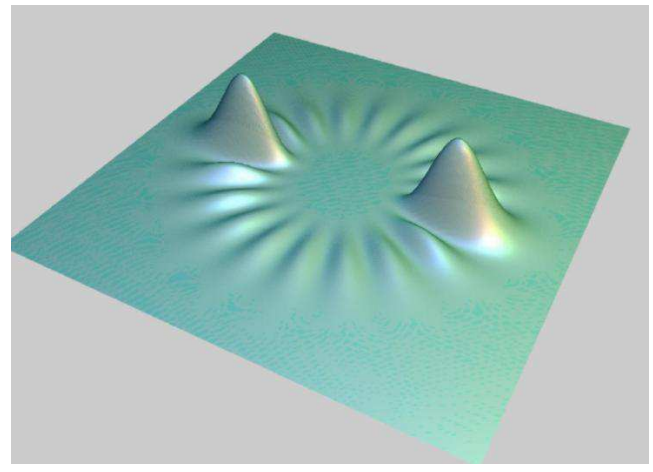

(b)

FIG. 1: (color online.) Plots of the radial probability density $r^{2}|\psi(r, \theta, \phi)|^{2}$ for two (nonrelativistic) states with quantum numbers $n=10$ and $l=9$, but different magnetic quantum numbers. Figure (a) displays the density of the states with $|m|=9$, in the plane of constant polar angle $\theta=\pi / 2$. In space, the probability density of the electron in this maximum- $|m|$ state describes a circular shape, which is why circular states are being viewed as analogues of classical planetary motion in the quantum domain. However, this characteristic circular pattern is restricted to the extremal magnetic quantum numbers $(|m|=l)$. Figure (b) shows the radial probability density of the state with $m=0$, in the plane of constant azimuth $\phi=0$. The two maxima in the probability density are in the direction of the polar angles $\theta=0$ and $\theta=\pi$.

the asymptotics of quantum electrodynamic corrections as a function of the quantum numbers. Such results can for instance be useful in comparing different calculational approaches to these corrections, and for obtaining general expressions valid for arbitrary quantum numbers within a specific manifold of states. Certain asymptotic structures have recently been conjectured for radiative corrections [21, 22, 23].

Furthermore, the analysis of highly-excited (bare) atomic states is motivated by recent experiments: e.g., in [24], the evaluation of self energies of circular states of orbital quantum number $l \simeq 30$ was required. The consideration of quantum electrodynamic corrections for highly-excited states is also relevant, in part, to the analysis of quantum electrodynamic corrections to dielectronic recombination resonances 25].

In this work, we thus study radiative corrections for highly excited hydrogenic states. Specifically, after recalling basic facts about relativistic corrections in Sec. IIA we proceed to the study of radiative effects in Secs. IIB and IIC with an emphasis on the radiative decay width of circular states in Sec. IIC The main part of the investigation reported here is contained in IID, where a numerical calculation of Bethe logarithms for highly and very highly excited circular states is described (see Table \below). This investigation then leads to the asymptotic formulas for Bethe logarithms which yield a relative accuracy of $10^{-5}$ or better, for general states within a specified manifold. The self-energy corrections studied in Sec. IID are the by far dominant radiative corrections for circular Rydberg states. Conclusions are drawn in Sec. III This paper follows the usual convention for hydrogenic quantum numbers: the principal quantum number is denoted by $n$, the orbital angular momentum by $l$ and the total electron angular momentum by $j$. As is customary in the literature, $Z$ denotes the nuclear charge number of the hydrogenlike ion under consideration, and $\alpha$ is the fine-structure constant. Natural units $\left(\hbar=c=\epsilon_{0}=1\right)$ are used throughout the text.

\section{RELATIVISTIC AND RADIATIVE EFFECTS}

\section{A. Relativistic corrections}

We briefly recall some basic facts about relativistic corrections to highly-excited states in hydrogenlike systems. The numerical values of the coefficients multiplying the relativistic corrections of order $(Z \alpha)^{4} m c^{2}$ are typically small for high- $n$ states. In order to illustrate this point, we briefly recall the expansion of the Dirac bound-state energy in a hydrogenlike system in powers of $Z \alpha$ up to order $(Z \alpha)^{4}$ (see, e.g., [26, Eq. (2-87)]):

$$
E_{n j}=m-\frac{(Z \alpha)^{2} m}{2 n^{2}}-\frac{(Z \alpha)^{4} m}{n^{3}}\left[\frac{1}{2 j+1}-\frac{3}{8 n}\right]+\mathcal{O}(Z \alpha)^{6}
$$

The $(Z \alpha)^{4}$-term is the dominant relativistic correction for low- $Z$ hydrogenlike systems and is responsible, in particular, for the fine structure. By inspection of Eq. (11), we infer that for circular states (which have $j=n-1 \pm 1 / 2$ ), the 
$(Z \alpha)^{4}$-term scales as $j^{-4} \sim n^{-4}$, which contrasts with the familiar $n^{-3}$-scaling valid for fixed $j$. As we will see below, radiative corrections in Rydberg states also tend to display faster asymptotic behaviors (in $n$ ) than states with a fixed angular momentum.

\section{B. Self Energy and Radiative Lifetime}

In this article, we study radiative effects in highly-excited hydrogenic energy levels. Our focus is on the self-energy and the radiative lifetime. As pointed out in Ref. 27, the self-energy and the radiative lifetime are intimately related to each other. Namely, the radiative lifetime of a hydrogenic state is proportional to the imaginary part of the expectation value of that state $\left\langle\psi\left|\Sigma_{\text {ren }}\right| \psi\right\rangle$, where $\Sigma_{\text {ren }}$ is the renormalized self-energy operator discussed in Ch. 7 of [26], and $\psi$ is the relativistic (Dirac) wave function. The self-energy is just the real part of $\left\langle\psi\left|\Sigma_{\text {ren }}\right| \psi\right\rangle$ and leads to the familiar shift of the energy levels.

As is well known, the real part of the one-loop self-energy shift $\Delta E_{\mathrm{SE}}$ may be written as

$$
\Delta E_{\mathrm{SE}}=\operatorname{Re}\left\langle\psi\left|\Sigma_{\mathrm{ren}}\right| \psi\right\rangle=\frac{\alpha}{\pi} \frac{(Z \alpha)^{4} m}{n^{3}} F\left(n l_{j}, Z \alpha\right) .
$$

Here, $F\left(n l_{j}, Z \alpha\right)$ is a dimensionless quantity, and the the notation $n l_{j}$ follows the usual spectroscopic characterization of the hydrogenic state $\psi$. It is customary in the literature to suppress the dependence of $F$ on the quantum numbers $n, j$ and $l$ and write $F(Z \alpha)$ for $F\left(n l_{j}, Z \alpha\right)$.

The decay width $\Gamma$ is the reciprocal of the lifetime $\tau$ and is given by

$$
\Gamma=\frac{1}{\tau}=-2 \operatorname{Im}\left\langle\psi\left|\Sigma_{\text {ren }}\right| \psi\right\rangle
$$

For states with the same angular momentum quantum numbers, the decay rate decreases with $n$ approximately as $n^{-3}$, and the lifetime correspondingly goes as $n^{3}$ (see Ref. [9]). This is also manifest in the definition (2) of the scaled self-energy function $F\left(n l_{j}, Z \alpha\right)$, the structure of which reflects the usual, familiar scaling of the effect with $n$ (for fixed $l$ and $j)$.

\section{Radiative Lifetime: Asymptotics for Rydberg States}

To a good approximation, the lifetime of a highly-excited hydrogenic state does not depend on the spin of the electron, and it is only the principal quantum number and the orbital angular momentum which enter into the leading-order (nonrelativistic) expression for the decay rate of excited hydrogenic states.

We denote the (leading-order) decay width of a state with principal quantum number $n$ and orbital angular momentum $l$ by the symbol $\Gamma(n, l)$. Here, we are particularly interested in states with $n=l+1$, and our goal is to study the dependence of the lifetime on the bound-state quantum numbers. For the manifold of states with $n=l+1$, based on the formalism introduced in [27], we obtain the following general expression,

$$
\begin{aligned}
\Gamma(n, n-1)= & \frac{2 \alpha}{3} \frac{(Z \alpha)^{4} m}{n^{5}}\left\{\frac{n^{2 n+1}(n-1)^{2 n-2}}{(n-1 / 2)^{4 n-1}}\right\} \\
= & \frac{2 \alpha}{3} \frac{(Z \alpha)^{4} m}{n^{5}} \times\left\{1+\frac{1}{n}+\frac{7}{8 n^{2}}+\frac{35}{48 n^{3}}\right. \\
& \left.+\frac{229}{384 n^{4}}+\frac{233}{480 n^{5}}+\mathcal{O}\left(n^{-6}\right)\right\} .
\end{aligned}
$$

The asymptotic expansion (4b) about large $n$ works surprisingly well, even at low principal quantum number. For $n=2$, the first few terms listed in (4b) reproduce the exact formula 4a to within $5 \%$ accuracy. For $n=40$, the difference is less than $10^{-10}$ in relative units. Equation (4b) implies that the lifetime of circular Rydberg states with $l=n-1$ scales effectively as $n^{5}$, in contrast to the familiar $n^{3}$-scaling which is valid for fixed $l$, but varying $n$.

Based on the well-behaved asymptotic structure of the leading non-relativistic contribution to $\operatorname{Im}\left\langle\psi\left|\Sigma_{\text {ren }}\right| \psi\right\rangle$ for high quantum numbers, we now turn our attention to the real part $\operatorname{Re}\left\langle\psi\left|\Sigma_{\text {ren }}\right| \psi\right\rangle$. 


\section{Self-Energy}

Vacuum-polarization effects are negligible for high-l circular Rydberg states [the radial component of the wave function scales as $(Z \alpha m r)^{l}$ for small $r$. The dominant radiative correction to hydrogenic energy levels is given by the (one-photon) self energy

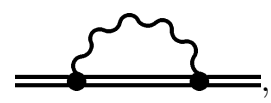

which is a process in which the bound electron (double line) emits and re-absorbs a photon (wavy line); this effect shifts the energies predicted by the Dirac equation.

We now turn to the investigation of the energy shift $\Delta E_{\mathrm{SE}}$, which is defined in Eq. (2) and corresponds to the above diagram. The semi-analytic expansion of $F\left(n l_{j}, Z \alpha\right)$ about $Z \alpha=0$ for a general atomic state with quantum numbers $n, l$ and $j$ gives rise to the expression [28]

$$
F\left(n l_{j}, Z \alpha\right)=A_{41}\left(n l_{j}\right) \ln \left[(Z \alpha)^{-2}\right]+A_{40}\left(n l_{j}\right)+\mathcal{O}(Z \alpha)
$$

The $Z \alpha$-expansion is semi-analytic, i.e., it involves powers of $Z \alpha$ and of $\ln \left[(Z \alpha)^{-2}\right]$. The $A$ coefficients have two indices, the first of which denotes the power of $Z \alpha$ [including those powers contained in Eq. (2)], while the second index denotes the power of the logarithm $\ln \left[(Z \alpha)^{-2}\right]$.

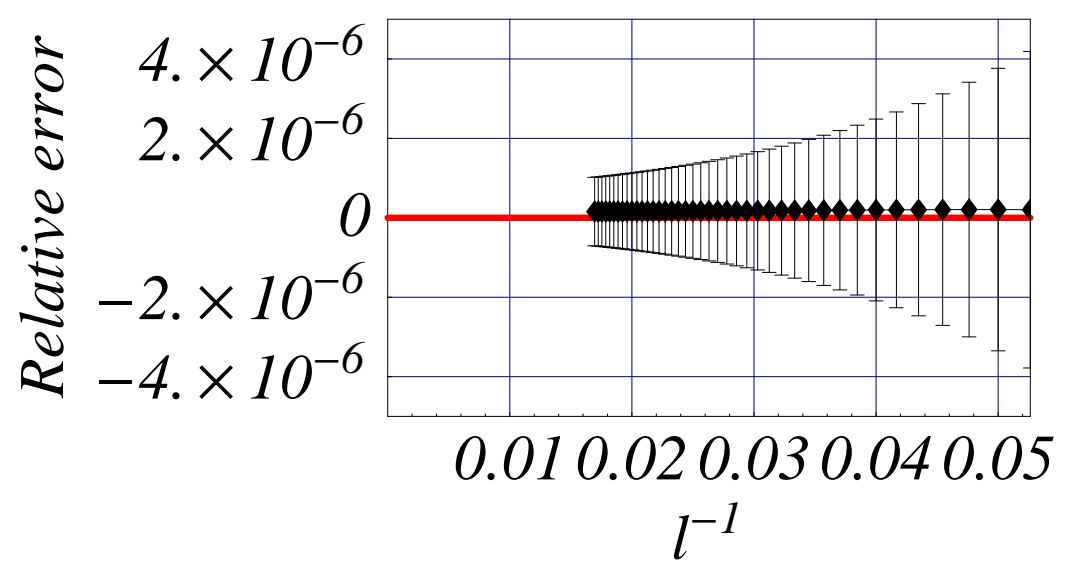

FIG. 2: (color online.) Relative difference between the Bethe logarithm values presented in Tab. 【 and the asymptotic formula (9), for circular Rydberg states $(l=n-1)$ in the range $20<n \leq 60$. Since zero is contained in all the error bars, the Bethe logarithms in Tab. I are fully consistent with the approximation (9). The error bars in the figure correspond to the uncertainty in the numerical coefficients of Eq. (9).

The coefficient $A_{41}\left(n l_{j}\right)$ assumes a value of $4 / 3$ for $S$ states and vanishes for all non- $S$ states. The general formula for $A_{40}$ reads (see, e.g., [29, 30])

$$
A_{40}=\frac{10}{9} \delta_{l 0}-\frac{1-\delta_{l 0}}{2 \kappa(2 l+1)}-\frac{4}{3} \ln k_{0}(n, l),
$$

where $\kappa=2(l-j)(j+1 / 2)$. The Bethe logarithm $\ln k_{0}(n, l)$ is an inherently nonrelativistic quantity, whose expression in natural units reads

$$
\begin{aligned}
& \ln k_{0}(n, l)=\frac{n^{3}}{2(Z \alpha)^{4} m} \\
& \times\left\langle\phi\left|\frac{p^{i}}{m}\left(H_{\mathrm{S}}-E_{n}\right) \ln \left[\frac{2\left|H_{\mathrm{S}}-E_{n}\right|}{(Z \alpha)^{2} m}\right] \frac{p^{i}}{m}\right| \phi\right\rangle .
\end{aligned}
$$


Here, $H_{\mathrm{S}}$ is the nonrelativistic Coulomb Schrödinger Hamiltonian, $p^{i}$ is the $i$ th Cartesian component of the momentum operator, $E_{n}$ and $\phi$ are respectively the (nonrelativistic) energy and the wave-function of a state with the quantum numbers $(n, l)$, and the summation over Cartesian coordinates $(i=1,2,3)$ is implicit.

Bethe logarithms have been studied for all hydrogenic states with $n \leq 20$, by various authors and with increasing accuracy due to advances in the algorithms used and in the computing technology available 20, 31, 32, 33, 34, 35, 36, 37, 38]. While the calculational difficulties (numerical convergence properties of the involved hypergeometric functions) increase as the principal quantum number increases, the evaluation of the hydrogenic nonrelativistic Bethe logarithm can be regarded as an easy computational task from the perspective of our current understanding of bound states, and of modern computer technology. In the current work, we employ the technique previously discussed in [23, 39], where the relevant matrix element of the hydrogenic wavefunction [1] is expressed in terms of hypergeometric functions ${ }_{2} F_{1}$, which are then suitably integrated. For high energies of the virtual photon, we found it useful to employ the combined nonlinear-condensation transformation [40, 42] in order to accelerate the convergence of the series representation of these hypergeometric functions.

One of the results we have obtained reads

$$
\ln k_{0}(n=20, l=19)=-0.000008084977837087891(1),
$$

a value which confirms the result obtained previously in Ref. [20] for this state. The error is due to the uncertainty in the final integration over the virtual photon energy. While the numerical accuracy of this result is merely of academic interest, we found it useful to verify the accuracy of our computational method against the basis-set techniques employed in $[20,38]$.

TABLE I: Values of the Bethe logarithm for circular Rydberg states (for which $l=n-1$ ), in the range $20<n \leq 60$. According to Eqs. (5) and (6), the Bethe $\operatorname{logarithm} \ln k_{0}$ contributes significantly to the radiative correction to the energy of hydrogenic states. All explicit results found here agree with a previously published expansion [23, Eq. (37)], as well as with the new asymptotic expansion (9), as is shown in Fig. 2 (within the numerical uncertainties in the asymptotic coefficients).

\begin{tabular}{ccccc}
\hline \hline$n$ & \multicolumn{1}{c}{$\ln k_{0}(n, n-1)$} & $n$ & & $\ln k_{0}(n, n-1)$ \\
\hline 21 & $-0.6941065660642(1) \times 10^{-5}$ & 41 & $-0.0878281749454(1) \times 10^{-5}$ \\
22 & $-0.6003118198591(1) \times 10^{-5}$ & 42 & $-0.0815806567501(1) \times 10^{-5}$ \\
23 & $-0.5226798437374(1) \times 10^{-5}$ & 43 & $-0.0759118687638(1) \times 10^{-5}$ \\
24 & $-0.4578744770701(1) \times 10^{-5}$ & 44 & $-0.0707563248884(1) \times 10^{-5}$ \\
25 & $-0.4033528104402(1) \times 10^{-5}$ & 45 & $-0.0660572331309(1) \times 10^{-5}$ \\
26 & $-0.3571536036972(1) \times 10^{-5}$ & 46 & $-0.0617651782987(1) \times 10^{-5}$ \\
27 & $-0.3177478119539(1) \times 10^{-5}$ & 47 & $-0.0578370279429(1) \times 10^{-5}$ \\
28 & $-0.2839314416323(1) \times 10^{-5}$ & 48 & $-0.0542350198720(1) \times 10^{-5}$ \\
29 & $-0.2547477154839(1) \times 10^{-5}$ & 49 & $-0.0509259980293(1) \times 10^{-5}$ \\
30 & $-0.2294298276714(1) \times 10^{-5}$ & 50 & $-0.0478807701395(1) \times 10^{-5}$ \\
31 & $-0.2073583589839(1) \times 10^{-5}$ & 51 & $-0.0450735657214(1) \times 10^{-5}$ \\
32 & $-0.1880292605357(1) \times 10^{-5}$ & 52 & $-0.0424815771656(1) \times 10^{-5}$ \\
33 & $-0.1710295447205(1) \times 10^{-5}$ & 53 & $-0.0400845698251(1) \times 10^{-5}$ \\
34 & $-0.1560186575648(1) \times 10^{-5}$ & 54 & $-0.0378645496597(1) \times 10^{-5}$ \\
35 & $-0.1427140813819(1) \times 10^{-5}$ & 55 & $-0.0358054790517(1) \times 10^{-5}$ \\
36 & $-0.1308801169928(1) \times 10^{-5}$ & 56 & $-0.0338930330782(1) \times 10^{-5}$ \\
37 & $-0.1203190769194(1) \times 10^{-5}$ & 57 & $-0.0321143898736(1) \times 10^{-5}$ \\
38 & $-0.1108643219585(1) \times 10^{-5}$ & 58 & $-0.0304580498130(1) \times 10^{-5}$ \\
39 & $-0.1023747182006(1) \times 10^{-5}$ & 59 & $-0.0289136791317(1) \times 10^{-5}$ \\
40 & $-0.0947301966836(1) \times 10^{-5}$ & 60 & $-0.0274719743302(1) \times 10^{-5}$ \\
\hline \hline
\end{tabular}

In Tab. I we present accurate numerical values for the Bethe logarithm (77) of circular Rydberg states, for $21 \leq$ $n \leq 60$. These values are in agreement with the truncated asymptotic expansion found in Ref. [23, Eq. (37)]. This asymptotic expansion had been derived on the basis of numerical data reported in Ref. [20], where the range $1 \leq n \leq 20$ had been covered. Based on the numerical data of Tab. [ which cover a wider range of principal quantum numbers as compared to Ref. [20], we obtain the following improved asymptotics for the Bethe logarithm of circular Rydberg states:

$$
l^{3} \times \ln k_{0}(l+1, l) \simeq-0.05685281(3)+\frac{0.0248208(6)}{l}+\frac{0.03814(2)}{l^{2}}-\frac{0.1145(5)}{l^{3}}+\frac{0.166(3)}{l^{4}}-\frac{0.22(2)}{l^{5}},
$$


where terms of order $l^{-k}$ with $k \geq 6$ are neglected. The algorithm described in the Appendix of [23] was used for obtaining the coefficients of this asymptotic expansion. All the coefficients in (9) separately are in very good agreement with those found previously 23, Eq. (37)]. The Bethe logarithm values in Tab. [1 are fully consistent with the above truncated expansion: they fall inside the error bars of (9), as is illustrated in Fig. 22 Furthermore, the "expectation" values for the coefficients of formula (9) correctly "predict" about seven digits of the actual numerical value of the Bethe logarithm in Tab. [1 as illustrated in Fig. 2] Based on the very good consistency of the asymptotic expansion with the actual numerical data (Fig. 22), we conjecture that the "expectation" values of the expansion (2) give the Bethe logarithm of all circular Rydberg states with $n>20$ with a relative precision better than $10^{-6}$, as can be expected from Fig. 22 Furthermore, we would like to conjecture here that the expansion (9) not only represents a "polynomial fit" to the numerical data, but that it represents the true asymptotic expansion of the Bethe logarithm for high quantum numbers.

For $S$ states, based on data available for principal quantum numbers $n \leq 20$ (see Ref. [20]), we obtain the following asymptotic expansion as a function of the principal quantum number $n$,

$$
\ln k_{0}(n, l=0) \simeq 2.72265434(5)+\frac{0.000000(5)}{n}+\frac{0.55360(5)}{n^{2}}-\frac{0.5993(5)}{n^{3}}+\frac{0.613(7)}{n^{4}}-\frac{0.60(5)}{n^{5}},
$$

where terms of order $n^{-k}$ with $k \geq 6$ are neglected. By a reasoning similar to the one above, we expect the expansion (10), with the "expectation" values employed for the coefficients, to give the Bethe logarithm of all $S$ states with $n>10$ to better than $2 \cdot 10^{-7}$ in relative units.

Finally, for high- $n P$ states, we obtain

$$
\ln k_{0}(n, l=1) \simeq-0.0490545(1)+\frac{0.000000(5)}{n}+\frac{0.20530(15)}{n^{2}}-\frac{0.599(5)}{n^{3}}+\frac{1.45(10)}{n^{4}}-\frac{3(1)}{n^{5}} .
$$

where again terms of order $n^{-k}$ with $k \geq 6$ are neglected. We expect Eq. (11) to reproduce the exact Bethe Logarithms of all $P$ states for $n>10$ to a relative accuracy better than $5 \cdot 10^{-5}$.

\section{CONCLUSIONS}

We have studied relativistic and radiative corrections for highly-excited hydrogenic states. In addition to $S$ and $P$ states, we have concentrated on circular Rydberg states (for which $l=n-1$ ). We have observed on the relativistic and radiative corrections considered here that circular Rydberg state corrections have a faster asymptotic behavior (as a function of $n$ ) than states with a fixed angular momentum.

The following scaling properties hold for the dominant relativistic and radiative effects on circular hydrogenic Rydberg states: (i) The leading relativistic corrections to the energy levels (of order $(Z \alpha)^{4} m c^{2}$ ) scale as $n^{-4}$. (ii) Radiative decay rates scale as $n^{-5}$ [see Eq. (4b]). (iii) The Bethe logarithm contribution to the self-energy shift (21) scales as $n^{-6}$. Note the three inverse powers of $l$ in Eq. (9) which have to be taken into consideration in addition to the three inverse powers of $n$ originating from (2). By contrast, the Bethe logarithm contribution for highly-excited $S$ and $P$ states scales as $n^{-3}$ [see Eqs. [10) and [11]. (iv) The anomalous magnetic moment contribution to the Lamb shift [which corresponds to the term $-\frac{1}{2 \kappa(2 l+1)}$ in Eq. (6)] scales as $n^{-5}$ for circular Rydberg states and thus dominates over the Bethe logarithm in the limit of large $l=n-1$.

In this article, we also provide an exact result for the decay rate of highly-excited hydrogenic Rydberg states [see Eq. (4a)]. Furthermore, we obtain accurate values (see Tab. (II) for the Bethe logarithms of circular Rydberg states (for which the orbital angular quantum number $l$ is related to the principal quantum number as $l=n-1$ ). These values are in a range of quantum numbers $21 \leq n \leq 60$, where no such data have been available up to now, to the best of our knowledge. These results confirm that the dependence of radiative corrections on the bound-state quantum numbers can be represented, to a very good accuracy, by asymptotic expansions about large $n$ which involve negative integer powers [see Eqs. (4b), (9), (10) and (11)]. This functional dependence has recently been observed for a number of QED effects [21, 22, 23]. We conjecture here that the Bethe logarithm expansions (91), (10) and (11) represent the true asymptotic expansions about large quantum numbers (to the precision indicated in the text).

The results obtained here are relevant for any analysis (e.g., [24]) where the evaluation of the self energy of circular $(n=l+1)$ states of orbital quantum number $l>20$ is required. They are also relevant for the description of the "bare" Lamb shift contribution to the Lamb shift of laser-dressed states 10, 11, 12, 14] in experiments with Rydberg states. A possible experimental setup would involve the metastable $2 S$ state and an excitation to a high- $n P$ state. The required frequency is smaller than the one required for the excitation $1 S \Leftrightarrow 2 P_{j}$, which has been studied in detail in [12]. Moreover, both states involved in the transition $2 S \Leftrightarrow n P_{j}$ (high $n$ ) have a long lifetime, which would facilitate the observation of the dressed-state corrections to the Lamb shift [10, 11, 12]. The formalism required for 
the analysis of further corrections to the laser-dressed Lamb shift has recently been described in 12 . The additional corrections are due to ionization, counter-rotating terms (Bloch-Siegert shifts, see [43]) and nonresonant levels, as well as laser-field configuration dependent effects.

Finally, we note that an accurate investigation of the Lamb shift of the (numerous) different magnetic subcomponents of circular Rydberg levels would allow for a sensitive test of the recently proposed, somewhat speculative "polarized Lamb shift" (see Ref. 44]) that predicts a breaking of the rotational symmetry imposed on quantum electrodynamics.

\section{Acknowledgments}

U.D.J. and E.O.L. acknowledge support from the National Institute of Standards and Technology during a number of research appointments. P. Indelicato is acknowledged for helpful discussions. J. Evers acknowledges support from the National German Academic Foundation.

[1] R. G. Hulet and D. Kleppner, Phys. Rev. Lett. 51, 1430 (1983).

[2] F. Roussel, M. Cheret, L. Chen, T. Bolzinger, G. Spiess, J. Hare, and M. Gross, Phys. Rev. Lett. 65, 3112 (1990).

[3] P. Nussenzveig, F. Bernardot, M. Brune, J. Hare, J. M. Raimond, S. Haroche, and W. Gawlik, Phys. Rev. A 48, 3991 (1993).

[4] M. Brune, F. Schmidt-Kaler, A. Maali, J. Dreyer, E. Hagley, J. M. Raimond, and S. Haroche, Phys. Rev. Lett. 76, 1800 (1996).

[5] E. Hagley, X. Maître, G. Nogues, C. Wunderlich, M. Brune, J. M. Raimond, and S. Haroche, Phys. Rev. Lett. 79, 1 (1997).

[6] I. Bialynicki-Birula, M. Kalinski, and J. H. Eberly, Phys. Rev. Lett. 73, 1777 (1994).

[7] J. Zakrzewski, D. Delande, and A. Buchleitner, Phys. Rev. Lett. 75, 4015 (1995).

[8] E. A. Shapiro, M. Kalinski, and J. H. Eberly, Opt. Express 3, 124 (1998).

[9] H. A. Bethe and E. E. Salpeter, Quantum Mechanics of One- and Two-Electron Atoms (Springer, Berlin, 1957).

[10] U. D. Jentschura, J. Evers, M. Haas, and C. H. Keitel, Phys. Rev. Lett. 91, 253601 (2003).

[11] U. D. Jentschura and C. H. Keitel, Ann. Phys. (N.Y.) 310, 1 (2004).

[12] J. Evers, U. D. Jentschura, and C. H. Keitel, Relativistic and Radiative Corrections to the Mollow Spectrum, e-print quant-ph/0403202

[13] K. S. E. Eikema, J. Walz, and T. W. Hänsch, Phys. Rev. Lett. 86, 5679 (2001).

[14] B. R. Mollow, Phys. Rev. 188, 1969 (1969).

[15] By contrast, direct transitions among Rydberg states are probably not a suitable environment for the study of the dressed Lamb shift in an intense laser field: the problem is that a laser which drives a circular Rydberg transition between levels with $n, l \Leftrightarrow n+1, l+1$ is approximately resonant not only with the desired transitions, but also with the transition $n+1, l+1 \Leftrightarrow n+2, l+2$, in the limit of large $n$. Therefore, the level scheme used in the analysis of the laser-dressed Lamb shift would have to be substantially generalized in order to accomodate for the many (near-resonant) energy levels.

[16] V. I. Korobov and S. V. Korobov, Phys. Rev. A 59, 3394 (1999).

[17] G. W. F. Drake and S. P. Goldman, Can. J. Phys. 77, 835 (1999).

[18] V. I. Korobov, Phys. Rev. A 69, 054501 (2004).

[19] Z.-C. Yan and G. W. F. Drake, Phys. Rev. Lett. 91, 113004 (2003).

[20] G. W. F. Drake and R. A. Swainson, Phys. Rev. A 41, 1243 (1990).

[21] U. D. Jentschura, E.-O. Le Bigot, P. Indelicato, P. J. Mohr, and G. Soff, Phys. Rev. Lett. 90, 163001 (2003).

[22] U. D. Jentschura, J. Phys. A 36, L229 (2003).

[23] E.-O. Le Bigot, U. D. Jentschura, P. J. Mohr, P. Indelicato, and G. Soff, Phys. Rev. A 68, 042101 (2003).

[24] J. C. deVries, Ph.D. thesis, Massachusetts Institute of Technology, Cambridge, MA, 2002.

[25] E. Lindroth, H. Danared, P. Glans, Z. Pesic, M. Tokman, G. Vikor, and R. Schuch, Phys. Rev. Lett. 86, 5027 (2001).

[26] C. Itzykson and J. B. Zuber, Quantum Field Theory (McGraw-Hill, New York, NY, 1980).

[27] R. Barbieri and J. Sucher, Nucl. Phys. B 134, 155 (1978).

[28] G. W. Erickson and D. R. Yennie, Ann. Phys. (N.Y.) 35, 271 (1965).

[29] J. Sapirstein and D. R. Yennie, in Quantum Electrodynamics, Vol. 7 of Advanced Series on Directions in High Energy Physics, edited by T. Kinoshita (World Scientific, Singapore, 1990), pp. 560-672.

[30] P. J. Mohr and B. N. Taylor, Rev. Mod. Phys. 72, 351 (2000).

[31] H. A. Bethe, Phys. Rev. 72, 339 (1947).

[32] H. A. Bethe, L. M. Brown, and J. R. Stehn, Phys. Rev. 77, 370 (1950).

[33] J. M. Harriman, Phys. Rev. 101, 594 (1956). 
[34] C. Schwartz and J. J. Tieman, Ann. Phys. (N.Y.) 6, 178 (1959).

[35] M. Lieber, Phys. Rev. 174, 2037 (1968).

[36] R. W. Huff, Phys. Rev. 186, 1367 (1969).

[37] S. Klarsfeld and A. Maquet, Phys. Lett. B 43, 201 (1973).

[38] S. P. Goldman and G. W. F. Drake, Phys. Rev. A 61, 052513 (2000).

[39] U. D. Jentschura and K. Pachucki, Phys. Rev. A 54, 1853 (1996).

[40] U. D. Jentschura, P. J. Mohr, G. Soff, and E. J. Weniger, Comput. Phys. Commun. 116, 28 (1999).

[41] R. A. Swainson und G. W. F. Drake, J. Phys. A 24, 79, 95, 1801 (1991).

[42] S. V. Aksenov, M. A. Savageau, U. D. Jentschura, J. Becher, G. Soff, and P. J. Mohr, Comput. Phys. Commun. 150, 1 (2003).

[43] F. Bloch and A. Siegert, Phys. Rev. 57, 522 (1940).

[44] M. Chaichian, M. M. Sheikh-Jabbari, and A. Tureanu, Phys. Rev. Lett. 86, 2716 (2001). 